\title{
Möglichkeiten und Gefahren der Meinungsfreiheit
}

Eine inhaltsanalytische Untersuchung der Diskussion in

deutschen überregionalen Tageszeitungen während des Karikaturenstreits 2006

\author{
Teresa K. Naab • Helmut Scherer
}

Die Originalpublikation ist 2009 erschienen in Publizistik, Vierteljahreshefte für Kommunikationsforschung, 54. Jahrgang, Heft 3, Seiten 373-389. Sie ist erhältlich unter www.springerlink.com.

\section{Korrespondenzanschrift:}

T. K. Naab M.A. ( $\square)$

Institut für Journalistik und Kommunikationsforschung, Hochschule für Musik und Theater Hannover, Expo Plaza 12, 30539 Hannover, Deutschland

E-Mail: teresa.naab@ijk.hmt-hannover.de 


\section{Möglichkeiten und Gefahren der Meinungsfreiheit}

Eine inhaltsanalytische Untersuchung der Diskussion in deutschen überregionalen Tageszeitungen während des Karikaturenstreits 2006

Zusammenfassung: Einer Debatte über die Meinungsfreiheit, wie sie vor dem Hintergrund des Karikaturenstreits im Jahr 2006 geführt wurde, kommt in der Demokratie besondere Bedeutung zu, da Meinungsfreiheit ein konstituierendes Element der Demokratie darstellt. Der Artikel untersucht, wie über die Möglichkeiten und Gefahren der Meinungsfreiheit in deutschen Zeitungen während des Karikaturenstreits diskutiert wurde. Dazu wurde eine quantitative Inhaltsanalyse der Argumente zum Thema in der Frankfurter Allgemeinen Zeitung, der Süddeutschen Zeitung und der tageszeitung durchgeführt. Nur in geringem Umfang fand eine vielseitige Diskussion über die Meinungs- und Medienfreiheit statt. Zwar stellten die Zeitungen ihre Grenzen ausführlich in Frage, unterstrichen ihre Schutzwürdigkeit und informierten über Bedrohungen; die Gewinne, welche die Meinungsfreiheit bietet, waren argumentativ jedoch kaum präsent. Eine Abwägung von Schranken und Chancen erfolgte hauptsächlich durch Medienakteure, die in dem Konflikt nicht nur Vermittler, sondern auch Beteiligte waren. Diese Ergebnisse führen zu einem Fazit über die Beachtung der Meinungsfreiheit als Kernwert der Demokratie.

Schlüsselwörter: Meinungsfreiheit $\bullet$ Demokratie $\bullet$ Argumentationsanalyse 


\title{
Chances and dangers of freedom of speech
}

\section{A content analysis of the discussion of Muhammad cartoons in German national dailies}

\begin{abstract}
A discussion about freedom of speech - such as the one arising from the publication of Muhammad caricatures in a Danish newspaper in 2006 - is of special importance in a democracy as it touches upon one of its elemental values. This article studies the debate on the value of freedom of speech as well as its potential dangers as it developed in the German newspapers after the publication of the caricatures. A quantitative content analysis was carried out encompassing all arguments posed in the Frankfurter Allgemeine Zeitung, Süddeutsche Zeitung and the tageszeitung. During the cartoon controversy, the discussion was only partly pluralistic. The limits to freedom of speech were elaborately put into question, the need to protect the freedom was highlighted, and threats to the freedom were covered. However, the benefits to be gained from a system of freedom of opinion were hardly present in the arguments put forth in the newspapers. An assessment of restraints or benefits mainly took place among the journalists themselves, who not only played the part of a messenger in the conflict, but were also an interested party in it. From these results, a conclusion is drawn on the consideration of freedom of speech as a core value of democracy.
\end{abstract}

Keywords: Freedom of speech $\bullet$ Democracy $\bullet$ Argumentation analysis 


\section{Einleitung}

Die Meinungsfreiheit scheint ein in Deutschland und in der westlichen Welt im Wesentlichen unangefochtenes Recht zu sein. Sie ist in der Erklärung der Menschenrechte der Vereinten Nationen festgeschrieben und in viele nationale Verfassungen aufgenommen. In der Bundesrepublik wird die Meinungs- und Medienfreiheit in Artikel 5 GG umfassend geschützt. Damit gehört sie zu den sogenannten Grundrechten, für die das Grundgesetz in Artikel 19 eine „Wesengehaltsgarantie“ vorsieht. Diese besagt, dass kein Grundrecht so eingeschränkt werden darf, dass es seine Aufgabe nicht mehr erfüllen kann. Tatsächlich ist aber die Meinungsfreiheit weder ungefährdet noch schrankenlos. Einschränkungen müssen immer dann diskutiert werden, wenn die Meinungsfreiheit in Konkurrenz zu anderen Grundrechten steht oder zu stehen scheint. So hat der Europäische Gerichtshof für Menschenrechte im sogenannten Caroline-von-Monaco-Urteil (vgl. EGMR 2004: 2647ff.) betont, dass die Pressefreiheit nicht rücksichtslos gegen andere Menschenrechte durchgesetzt werden darf.

Für solche Diskussionen und die Abwägung verschiedener Ansichten ist die Demokratie „ein unschätzbares Kampffeld [...], da sie Reformen ohne Gewaltanwendung zuläßt“ (Popper 1980: 198). Die Debatte erhält aber insofern besonderes Gewicht, als die zur Diskussion stehende Meinungsfreiheit ein konstituierendes Element der Demokratie selbst darstellt (vgl. Bundesverfassungsgericht 1975: 287). Eingriffe in die Meinungsfreiheit sind damit immer auch Eingriffe in das demokratische System insgesamt (vgl. Popper 1980: 198, 199). In diesem Bewusstsein und nach den Erfahrungen der Weimarer Republik verfolgt die Bundesrepublik das Konzept der wehrhaften oder auch streitbaren Demokratie. Nach diesem Streitbarkeitsprinzip können die wichtigsten Elemente der Demokratie auch durch eine noch so große Mehrheit nicht aufgehoben werden (zur Begründung der streitbaren Demokratie vgl. z.B. Bundesverfassungsgericht 
1952: 1; Denninger 1977; Eisel 1986; Hammans 1987). Der generelle und unaufhebbare Schutz des demokratischen Systems und der Meinungs- und Medienfreiheit ist jedoch als „kleinster gemeinsamer Nenner“ zu verstehen, dessen Interpretation notwendig umstritten bleibt (Hammans 1987: 120). Es muss eine ständige Abwägung zwischen den Gründen für die Meinungsfreiheit und ihren Begrenzungen stattfinden.

$\mathrm{Zu}$ untersuchen, wie in deutschen Medien über die Meinungsfreiheit diskutiert wird und wie zwischen ihren Vorteilen und Risiken abgewogen wird, ist Ziel dieses Artikels.

Dies geschieht vor dem Hintergrund des Karikaturenstreits 2005/06, der in Deutschland wie in vielen anderen Ländern eine umfangreiche Debatte über die Meinungsfreiheit auslöste und die fortwährende Aktualität einer Diskussion über die Grenzen der Meinungsfreiheit zeigt.

Die in einer dänischen Zeitung veröffentlichten islamkritischen Darstellungen des Propheten Mohammed wurden weltweit verbreitet (vgl. Debatin 2007a) und provozierten zum Teil heftige Reaktionen. Die Karikaturen bezogen sich unter anderem auf gewalttätigen islamischen Fundamentalismus und Frauendiskriminierung. Einige widmeten sich aber auch nicht direkt dem Islam, sondern kritisierten die Redaktion der verantwortlichen Zeitung Jyllands-Posten. Unterschiedliche Vorstellungen von Meinungsfreiheit und Religionskritik prallten aufeinander. Während die einen auf die Freiheit der Meinung als unverhandelbarem Grundwert der westlichen Gesellschaft und die Notwendigkeit von Kritik pochten, sahen andere die Würde einer Religionsgruppe sowie deren Handlungen herabgesetzt, verwiesen auf notwendige Schranken der Meinungs- und Medienfreiheit oder forderten zusätzliche Begrenzungen (einen detaillierten Überblick über den Karikaturenstreit bieten u.a. Debatin 2007b; Kunelius et al. 2007).

Es ist anzunehmen, dass der Schwerpunkt der Diskussion auf einer Abwägung zwischen Religion und Meinungsfreiheit lag. Allerdings dürfte der Karikaturenstreit auch zur 
Reflexion über andere Schranken und Begründungen für den Schutz des Grundrechts geführt haben. Eine quantitative Abbildung der Diskussionsinhalte und ihres Verlaufs vermag dazu beizutragen, die aktuelle Stellung der Meinungsfreiheit in Deutschland, ihre Wertschätzung und im Konfliktfall als relevant betrachtete Begrenzungen zu identifizieren: Welche Inhalte werden in der Diskussion angesprochen, und welche Argumentationsmuster finden sich?

Um mögliche Inhalte der Debatte zu erschließen, werden zunächst ideengeschichtliche Begründungen sowie rechtliche und ethische Beschränkungen der Meinungsfreiheit erläutert.

Die empirische Studie beschränkt sich auf eine Betrachtung der Diskussion in den Medien. Diese sind in der Debatte zweifach gefordert, weil Medien einerseits gesellschaftliche Entwicklungen widerspiegeln und die Diskussion andererseits ihre eigene Arbeitsgrundlage betrifft. Anschließend wird deshalb die Rolle der Medien in dem Konflikt erörtert und hergeleitet werden, inwiefern eine Konzentration der Diskursanalyse auf die Medienberichterstattung einen relevanten Einblick liefert.

\section{Begründungen und Schranken der Meinungsfreiheit}

Im Wesentlichen gibt es zwei zentrale Argumente für die Meinungsfreiheit (vgl. Milton 1984, erstmals 1644; Wilke 1987). Meinungsfreiheit ist ein individuelles, unveräußerliches Menschenrecht, denn Milton (1984) begreift den Menschen als vernunftbegabtes Wesen mit der Freiheit, zu wählen und zwischen Gut und Schlecht zu unterscheiden, wofür ihm unterschiedsfrei alle Informationen zur Verfügung stehen müssen. Milton führt außerdem ein kollektiv-soziologisches Argument an, das der Pressefreiheit einen überindividuellen, objektiven Nutzen zuschreibt. Die Wahrheitsfindung soll ein kumu- 
lativer und kollektiver Prozess sein, an dem sich alle beteiligen. Die Pressefreiheit nützt der Gesellschaft, die durch die in lebhafter Auseinandersetzung gewonnene Wahrheit erstarkt (vgl. Milton 1984). Auch das Bundesverfassungsgericht greift in seiner „Doppelbegründung“ (vgl. u.a. Grimm 1995: 1698) auf diese beiden Argumentationen zurück: „Das Grundrecht auf freie Meinungsäußerung ist als unmittelbarster Ausdruck der menschlichen Persönlichkeit in der Gesellschaft eines der vornehmsten Menschenrechte überhaupt [...]. Für eine freiheitlich-demokratische Staatsordnung ist es schlechthin konstituierend, denn es ermöglicht erst die ständige geistige Auseinandersetzung, den Kampf der Meinungen, der ihr Lebenselement ist [...].“ (Bundesverfassungsgericht 1958: 198)

Welche Bedeutung man der Meinungsfreiheit zuschreibt, ergibt sich aus den oben erläuterten Argumentationen: Wer die Meinungsfreiheit als individuelles Menschenrecht begründet, sieht in ihr eine Grundlage der menschlichen Würde und positive Folgen für die individuelle Entfaltung. Wer die kollektive Begründung wählt, erwartet „to help to discover truth, to assist in the process of solving political and social problems by presenting all manner of evidence and opinion as the basis for decision" (Siebert et al. 1963: 51). Unter der Freiheit der Meinungsäußerung kann Kritik an Obrigkeiten oder Missständen formuliert werden und als Kontrollinstanz für das politische System dienen. Die Vorstellung der Medien als vierte Gewalt im Staat basiert auf diesem Verständnis (vgl. Siebert et al. 1963: 51). Die der Presse in Deutschland durch das Bundesverfassungsgericht und die Landespressegesetze zugeschriebene öffentliche Aufgabe ist es deshalb, Sprachrohr zu sein, durch das sich die öffentliche Meinung artikuliert, als Motor die öffentliche Diskussion in Gang zu halten sowie Kontrolle und Kritik des politischen, wirtschaftlichen und kulturellen Geschehens zu leisten. Diese Aufgabe kann 
die Presse freilich wiederum nur in einem freien Mediensystem erfüllen, weshalb sie besonderen Schutz erhält (vgl. Ricker 2000: 246-247).

Eine Diskussion über die Meinungsfreiheit und ihre tatsächliche Ausgestaltung ist jedoch in der Demokratie nicht nur möglich, sondern notwendig; denn Presse- und Meinungsfreiheit gelten nicht unbeschränkt. In Deutschland haben sich Journalistenund Verlegerverbände im Deutschen Presserat auf den Pressekodex mit 16 Grundsätzen und angefügten Richtlinien verständigt, um die Berufsethik der Presse zu konkretisieren (vgl. Bölke 2000; Deutscher Presserat 2006; für eine Diskussion des Pressekodex im Hinblick auf die Karikaturen siehe Pöttker 2007). Ein Korrektiv für Fehlleistungen der Medien bildet die Rechtsprechung. Das Grundgesetz spricht von den allgemeinen Gesetzen und insbesondere vom Gesetz zum Schutze der Jugend und dem Recht der persönlichen Ehre; eine zentrale Schranke für die Meinungsfreiheit sind also die Grundrechte anderer Menschen. Bei solchen Grundrechtskonflikten muss es im Einzelfall immer wieder zu einer Abwägung zwischen dem Grundrecht der Meinungs- und Medienfreiheit und dem anderen durch das Gesetz geschützten Interesse kommen. Bei dieser Abwägung werden die allgemeinen Gesetze aufgrund der oben beschriebenen Bedeutung des Grundrechts durch dieses selbst wieder eingeschränkt, und es wird ein Ausgleich zwischen den kollidierenden Interessen hergestellt (vgl. Bundesverfassungsgericht 1958: 198; für einen Überblick über den Schutzumfang und die Schranken des Artikel 5 GG auf Grundlage der Entscheidungen des BVerfG vgl. Grimm 1995).

Im Konflikt um die Mohammed-Karikaturen trafen zwei Streitparteien aufeinander, von denen die eine einen unzulässigen Angriff auf eine Religionsgemeinschaft und die menschliche Würde beklagte und die andere sich für das Recht auf freie Meinungs- 
äußerung einsetzte. Hier bestand also enormer Diskussionsbedarf bei der Abgrenzung von Grundrechten (vgl. Amirthalingam 2007).

\section{Rolle der Medien in der Diskussion}

In einer pluralistischen Gesellschaft werden Auseinandersetzungen durch öffentliche Kommunikation ausgetragen. „Nur durch öffentliche Darstellung und Diskussion erlangen die Interessen demokratische Legitimation.“ (Ronneberger 1977: 14) Die folgenreichste Ebene der Öffentlichkeit ist die Massenkommunikation. Erst wenn Themen oder Meinungen in den Massenmedien aufgegriffen werden, erlangen sie allgemeine Wahrnehmung; denn räumliche, zeitliche und finanzielle Hindernisse können zugunsten hoher Reichweite überwunden werden. „Die öffentliche Sphäre ist deshalb mehr oder weniger in den Massenmedien institutionalisiert.“(Berens 2001: 20) Nach Weßler (1999: 74-75 und 222-223) übernehmen Journalisten dabei bis zu einem gewissen Grad die Rolle von Vermittlern in einem Konflikt, indem sie die Positionen der Kontrahenten gegeneinander abwägen und verschiedene Deutungen einander gegenüberstellen, während die anderen Akteure eher bestimmte Problemdeutungen betonen. Das heißt aber nicht, dass Journalisten nicht auch bestimmte Positionen vertreten können. Sie können selbst zu Sprechern in einem Konflikt werden, denn ihre Rolle ist nicht auf die der Bereitstellung eines Öffentlichkeitsforums für andere Akteure beschränkt. Zum Beispiel in Form von Kommentaren können sie sich explizit zum Sachverhalt äußern (vgl. Kepplinger 1994: 225). Außerdem geht es bei dem Karikaturenstreit zentral um die Rolle der Medien, diese sind direkt betroffen und damit selbst Konfliktakteure, weil die Meinungs- und Medienfreiheit ihre Arbeitsgrundlage darstellt. Die Abwägung zwischen Freiheit und Begrenzung ist also für die Medien von existenzieller Bedeutung. Dies dürfte ihre Rolle als Faktor in der Meinungsbildung verstärken (vgl. Mathes 1987: 166). 
Die vorliegende Studie konzentriert sich deshalb auf die Diskussion über die Meinungsfreiheit, wie sie in deutschen Zeitungen geführt wurde, da sie in der Berichterstattung sowohl gespiegelt als auch durch Medienakteure vorangetrieben werden dürfte. Ein Vergleich der Einlassungen von Medienakteuren mit denen anderer Diskursteilnehmer ermöglicht eine relationale Positionsbestimmung. Diese ist zwar auf den Karikaturenstreit beschränkt und von begrenzter Generalisierbarkeit, dürfte jedoch Anhaltspunkte für die grundsätzliche Sichtweise der beteiligten Akteure liefern. Eine Untersuchung von Zeitungen unterschiedlicher politischer Tendenz erscheint sinnvoll, um mögliche Unterschiede in der Berichterstattung und Kommentierung zu berücksichtigen. Über eine Feststellung der Diskussionsinhalte hinaus soll folglich analysiert werden: Welche Rolle spielen die Medien als Teilnehmer im Diskurs, und wie bringen sich andere Akteure ein?

\section{Methode}

Um die Inhalte der Diskussion über die Meinungsfreiheit und ihre Herkunft angemessen zu untersuchen, wurde das Verfahren der quantitativen Argumentationsanalyse gewählt (zum Verfahren der Argumentationsanalyse siehe Weiß [1989] und im Überblick [Weßler 1999]). Dabei wurden die in den Medien der Stichprobe auftauchenden Argumente inhaltsanalytisch erfasst. Als Argument galt dabei jede Aussage über die Meinungsfreiheit, die dieses Konstrukt ausdifferenziert, etwa durch eine Stellungnahme zur Notwendigkeit der Meinungsfreiheit, zu Einschränkungen oder ihrem Schutzumfang. Diese Ausdifferenzierung konnte sowohl neutral als auch wertend sein und musste nicht notwendigerweise selbst begründet werden. In Vorbereitung der Studie wurde nach theoretischer Vorarbeit eine qualitative Inhaltsanalyse von Hintergrundartikeln der Magazine Focus und Spiegel und der überregionalen Wochenzeitung Die Zeit zum Karikaturen- 
streit durchgeführt, um die Bandbreite der Argumentinhalte und Diskursteilnehmer zu fassen. Dabei zeigte sich, dass die Medien und die zitierten Akteure deutlich differenzierten, auf welchen kulturellen bzw. geografischen Rahmen ihre Argumente bezogen waren (Meinungsfreiheit in Deutschland, Dänemark, anderen westlich geprägten Ländern, der Türkei oder in islamisch geprägten Staaten). Dies wird deshalb in der quantitativen Analyse ebenfalls berücksichtigt.

Auf Basis dieser Dimensionierung erfolgte eine quantitative Inhaltsanalyse, bei der alle Argumente, die zur Meinungsfreiheit im Zusammenhang mit dem Karikaturenstreit auftauchten, ${ }^{1}$ deren Urheber und deren Bezugsrahmen erhoben wurden. Sofern sowohl Argumentinhalt, Argumenturheber als auch Bezugsrahmen identisch waren, wurde ein Argument pro Artikel nur einmal kodiert. Durch diese Festlegung ließ sich eine Häufung gleicher Argumente aufgrund eines wiederholenden Schreibstils vermeiden. ${ }^{2}$ Um die Meinung der Medien von der Meinung derjenigen abzugrenzen, über die sie berichten, und so die Rolle der Medien im Diskurs genauer bestimmen zu können, wurde die Stilform eines Artikels erhoben. Meinungsartikel werden als Ausdruck der Position der Medien verstanden.

Um den Diskurs in den deutschen Medien im Sample abzubilden, wurden drei überregionale Tageszeitungen in die Analyse einbezogen, die in Deutschland für ein breites publizistisches Spektrum auf hohem journalistischem Niveau bekannt sind und die als

\footnotetext{
${ }^{1}$ Die Abgrenzung der Begriffe Meinungs-, Presse- und Medienfreiheit wird nicht einheitlich vorgenommen und führt zu Missverständnissen (vgl. Marcic 1986: 6). Es ist deshalb zu erwarten, dass die Begriffe in den untersuchten Zeitungen synonym oder unsystematisch verwendet werden. Deshalb werden alle relevanten Argumente aufgegriffen, unabhängig davon, ob konkret von Meinungsfreiheit oder einem verwandten Begriff die Rede ist, um die Untersuchung der Diskussion nicht unangemessen einzuschränken und den Diskussionsbeiträgen gerecht werden.

${ }^{2}$ Bei einer Zusammenfassung von Dimensionen führt dies zu einer Verringerung der Argumentzahl (siehe Ergebnisteil, Abschnitt Positionen der Diskursteilnehmer), weil anfangs unterschiedliche Argumente dann zur gleichen Kategorie gehören und gleiche Argumente nur einmal gezählt werden.
} 
Leitmedien im deutschen Mediensystem gelten (vgl. Mathes/Czaplicki 1993). Um eine Bandbreite journalistischer Sichtweisen abzudecken, berücksichtigte die Auswahl unterschiedliche politische Linien. Die Frankfurter Allgemeine Zeitung gilt als gemäßigt rechts, die Süddeutsche Zeitung als gemäßigt links (vgl. Kepplinger 1998). Die tageszeitung repräsentiert wichtige alternative Meinungen (vgl. Mathes/Pfetsch 1991: 40). Ein Vergleich der Diskussionsbeiträge in den drei Zeitungen soll Hinweise auf die Unterschiedlichkeit der Debatte in der Zeitungsberichterstattung geben.

Der Untersuchungszeitraum reichte vom 30. September 2005 - dem Tag, an dem die Karikaturen in Dänemark erstmals veröffentlicht wurden - bis zum 31. März 2006. Die Intensität der Berichterstattung und die Kommentierung der Vorfälle ließen dann deutlich nach.

In der Analyse wurden alle Beiträge berücksichtigt, die sich im Zusammenhang mit den Mohammed-Karikaturen mit der Meinungs- und Medienfreiheit beschäftigten. Die Recherche der Artikel konnte mit Hilfe der digitalen Artikelsuche, die alle drei Zeitungen zur Verfügung stellten, durchgeführt werden. Aufgrund ihrer geringen Relevanz für die Diskussion um die Meinungsfreiheit wurden einige Ressorts aus der Analyse ausgeschlossen. Untersucht wurden nur Artikel auf Seiten für Politik, Kultur, Meinung, Medien, Zeitgeschehen und Panorama sowie Titelseiten. Leserbriefe, Pressespiegel, Bilder, eindeutig satirische Artikel und Ähnliches wurden nicht analysiert. Somit standen für die Analyse letztendlich 239 Artikel zur Verfügung, in denen mindestens ein Argument zur Meinungs- und Medienfreiheit vor dem Hintergrund des Karikaturenstreits vorkam. Das Kategoriensystem wurde vor der eigentlichen Untersuchung in einem Pretest erprobt und anschließend stellenweise überarbeitet. Die Werte für die Interkoderreliabilität waren zufriedenstellend, sie lagen für alle Variablen zwischen .7 und 1.0 (Verhältnis von übereinstimmenden Kodierungen zur Gesamtzahl der Kodierungen). 
In der Analyse werden die einzelnen Argumente getrennt voneinander erfasst, um eine detaillierte Auswertung einzelner inhaltlicher Aspekte der Meinungsfreiheit zu ermöglichen. Um Aussagen über die Gesamtaussage von Artikeln und die Argumentationsweisen in der Medienberichterstattung treffen zu können, ist es aber nötig, die tatsächlichen Argumentationsmuster zu untersuchen. Dazu müssen die inhaltlichen Kombinationen von Argumenten in den Artikeln betrachtet werden. Denn Argumente entfalten ihre Bedeutung erst im Lichte der anderen Argumente, mit denen sie gemeinsam vorgetragen werden. Um typische Argumentationsmuster zu erfassen, fiel die Wahl auf eine Auswertungsstrategie, die sich an der Framing-Forschung orientiert (vgl.

Matthes/Kohring 2004). Die Daten werden in der Form dichotomisiert, dass für jeden Artikel die Angabe vorliegt, ob ein Argumentinhalt mindestens einmal vorkam oder nicht. Sonstige Argumente bleiben unberücksichtigt, weil sich die dahinterstehende Aussage nicht mehr zuordnen lässt. Entsprechend werden Artikel, die nur sonstige Argumentinhalte enthalten, aus der Analyse ausgeschlossen. Mit Hilfe dieser neuen Variablen wird eine hierarchische Clusteranalyse (Distanzmaß: binäre quadrierte Euklidische Distanz; Ward-Verfahren zur Clusterbildung) durchgeführt, um Artikelgruppen mit ähnlichen Argumentationen zu identifizieren. Argumentationsstränge werden also nicht als Ganzes erhoben, sondern erst in der Datenauswertung aufgefunden.

\section{Ergebnisse}

\subsection{Inhalte des Mediendiskurses}

Insgesamt konnten 839 Aussagen verschiedener Akteure identifiziert werden (vgl. Tab. 1). Dabei fällt als Erstes auf: Gründe für die Meinungsfreiheit - kollektive wie individuelle - werden in nur $1 \%$ der Argumente angesprochen. Auch positive Folgen oder 
der Zweck der Meinungsfreiheit werden nur dreimal angesprochen. Auf die Bedeutung der Meinungsfreiheit (allgemein, für das Wertesystem, für die Demokratie oder anderes) gehen lediglich $4 \%$ der Aussagen ein. Damit wird im Karikaturenstreit über ein Grundrecht diskutiert, dieses aber kaum begründet und die juristische Grundlage der Meinungsfreiheit kaum erwähnt (1\%).

Am häufigsten wird über die Grenzen der Meinungs- und Medienfreiheit diskutiert, 42 $\%$ der Argumente beschäftigen sich damit. Es finden sich dabei deutlich häufiger Argumente, in denen eine Zulässigkeit bestimmter Beschränkungen festgestellt oder der Wunsch nach Schranken geäußert wird (26\% aller Argumente), als dass Journalisten generelle oder konkrete Begrenzungen der Meinungs- und Medienfreiheit ablehnen (12 $\%)$ oder keine Stellung zur Begrenzung der Meinungsfreiheit beziehen (1\%). Bei der Grenzdiskussion ist Religion das bestimmende Thema: $16 \%$ aller Argumente beziehen sich auf Religion als Schranke. Am häufigsten kommt die Position vor, Meinungs- und Medienfreiheit müsse oder solle hinter den Gefühlen von Gläubigen zurückstehen oder dürfe nicht dazu genutzt werden, um Religion oder Religionsgemeinschaften herabzusetzen. Auch die Verletzung von Vorschriften einer Religion (wie z.B. das islamische Bilderverbot) wird - allerdings deutlich seltener - kritisiert. Weitere Grenzen der Meinungs- und Medienfreiheit kommen zwar insgesamt häufiger vor (26\%), diese Argumente verteilen sich aber auf zahlreiche unterschiedliche inhaltliche Aspekte wie die Nutzung der Meinungsfreiheit zu Intoleranz, Fremdenfeindlichkeit und Volksverhetzung, Verletzung der Würde und Rechte von Menschen, Provokation oder die Verknüpfung ihrer Zulässigkeit mit der Forderung nach verantwortungsvollem Umgang. Nach den Grenz-Argumenten werden am zweithäufigsten Argumente zur möglichen oder tatsächlichen Bedrohung der Meinungs- und Medienfreiheit (16\%) genannt. Hier spiegeln sich die Geschehnisse des Karikaturenstreits - Zensurmaßnahmen (7 \%) und 
die Bedrohung von Medienschaffenden (5\%) - wider. $8 \%$ der Argumente diskutieren die generelle Schutzwürdigkeit der Meinungs- und Medienfreiheit unabhängig von bestimmten Grenzen. Die Gruppe der sonstigen Argumente ist mit $27 \%$ sehr groß. In ihr finden sich aber keine größeren inhaltlich ähnlichen Argumentgruppen, sondern nur zahlreiche Einzelargumente. Dies zeigt, dass diverse Aspekte in den Diskurs eingebracht wurden, die jedoch nicht von anderen Akteuren aufgegriffen wurden.

Tab. 1: Argumentinhalte zur Meinungsfreiheit (MF)

\begin{tabular}{lc}
\hline Argumentinhalte & in \% \\
\hline Grenze der MF & 42 \\
Bedrohung der MF & 16 \\
Schutzwürdigkeit der MF & 8 \\
Wichtigkeit der MF & 3 \\
Begründung der MF & 1 \\
Grundlage der MF & 1 \\
Folge/Zweck der MF & 0 \\
Bewertung der MF & 0 \\
Sonstige Argumentinhalte & 27 \\
Gesamt & 100 \\
\hline
\end{tabular}

$\mathrm{N}=839$ (Argumente)

Ausprägungen theoriegeleitet zusammengefasst

Um die Daten für die weiteren Analysen handhabbar zu machen, wurden Argumentinhalte, die in weniger als $5 \%$ aller Argumente vorkamen, theoriegeleitet zusammengefasst. Bei Argumenten zu Grenzen der Meinungs- und Medienfreiheit unterschieden wir weiterhin, ob Grenzen bestätigt, abgelehnt oder als unklar identifiziert wurden. Außer- 
dem betrachteten wir auf Religion bezogene Grenzen von anderen getrennt. (Zugunsten dieser Differenzierung waren hier Prozentanteile von unter 5 zugelassen.) ${ }^{3}$

\subsection{Positionen der Diskursteilnehmer}

Tab. 2 zeigt die Gruppen von Argumentinhalten nach ihren Urhebern. Am häufigsten äußern sich die Autoren der Artikel selbst (30\% der Argumente, $\mathrm{n}=220$ ), wobei zu beachten ist, dass eine Aussage, die keinem Urheber zugeordnet werden konnte, automatisch als Aussage der Zeitung galt. Des Weiteren werden für $10 \%$ der Argumente (n = 73) andere Medien zitiert. Argumente für die Begrenzung der Meinungsfreiheit nennen alle Akteursgruppen am häufigsten. Nur die Medienakteure argumentieren genauso häufig gegen eine Beschränkung der Meinungsfreiheit wie dafür. Sie diskutieren also zweiseitig, was die Schranken anbelangt. ${ }^{4}$ Die anderen Gruppen nennen deutlich weniger die Grenzen ablehnende Argumente. Politiker halten sich mit Ablehnungen von Schranken der Meinungsfreiheit zurück, betonen dafür ihre allgemeine Schutzwürdigkeit. Sie nehmen damit eine ausgleichende Haltung zwischen den Interessensgruppen ein: Einerseits stellen sie heraus, dass die Anliegen von Minderheiten geachtet werden müssen, andererseits verteidigen sie das Grundrecht generell. Auf eine Diskussion möglicher Grenzen ohne letztendliche Entscheidung verzichten sie im Gegensatz zu den anderen Gruppen völlig. Die Autoren der Artikel interessieren sich stärker für die Bedrohungen der Meinungsfreiheit als alle anderen Gruppen. Hierin unterscheiden sie sich deutlich von den in den untersuchten Zeitungen zitierten Medien. Dies dürfte daran

\footnotetext{
${ }^{3}$ Durch die Bündelung der Argumente reduzierte sich die Gesamtanzahl der Argumente von 839 auf 740 , weil ursprünglich unterschiedliche Argumente zu einer Kategorie zusammengefasst, per Definition aber gleiche Argumente nur einmal gezählt wurden.

${ }^{4}$ Auf dieser Ebene der Auswertung einzelner Argumente lässt sich nicht belegen, ob Pro- und KontraArgumente tatsächlich zusammen auftreten. Dazu ist eine Analyse auf Artikelebene notwendig (s.u.).
} 
liegen, dass es sich bei diesen Argumenten oft um Berichterstattung über konkrete Vorfälle wie die Bedrohung der Karikaturisten oder die Zensur von Karikaturnachdrucken handelt. Die Angabe anderer Medien als Quelle der reinen Berichterstattung erscheint den Journalisten vermutlich nicht nötig. Eher seltene Argumente (unter Sonstiges zusammengefasst) werden dagegen eher aus anderen Medien zitiert. Vereine und Organisationen äußern sich ebenfalls häufig zu Bedrohungen, räumen aber gleichzeitig die Notwendigkeit von Schranken ein. Dies kann darauf zurückgeführt werden, dass einige Nichtregierungsorganisationen wie „Reporter ohne Grenzen“ an der Diskussion teilnehmen, die sich vorrangig mit Bedrohungen der Meinungsfreiheit beschäftigen. 
Tab. 2: Argumentinhalte nach Urhebergruppen, in\%

\begin{tabular}{|c|c|c|c|c|c|c|}
\hline & $\begin{array}{l}\text { eigene } \\
\text { Zeitung, } \\
\text { Autor }\end{array}$ & $\begin{array}{l}\text { andere } \\
\text { Medien }\end{array}$ & $\begin{array}{l}\text { Politik, } \\
\text { polit. } \\
\text { Gruppe }\end{array}$ & $\begin{array}{l}\text { Verein, } \\
\text { NGO, } \\
\text { Institution }\end{array}$ & $\begin{array}{l}\text { Sonst. } \\
\text { Urheber- } \\
\text { gruppen }\end{array}$ & Gesamt \\
\hline Argumentinhalte & $(\mathbf{n}=\mathbf{2 2 0})$ & $(n=73)$ & $(\mathrm{n}=\mathbf{2 0 6})$ & $(n=41)$ & $(\mathrm{n}=\mathbf{2 0 0})$ & $(n=740)$ \\
\hline Befürwortung der Beschränkung der MF durch Religion ${ }^{a}$ & 4 & 8 & 17 & 22 & 23 & 14 \\
\hline Befürwortung der Beschränkung der MF durch eine sonstige Grenze ${ }^{a}$ & 9 & 10 & 16 & 15 & 14 & 13 \\
\hline Ablehnung der Beschränkung der MF durch Religion ${ }^{b}$ & 4 & 7 & 2 & - & 5 & 4 \\
\hline Ablehnung der Beschränkung der MF durch eine sonstige Grenze ${ }^{b}$ & 8 & 11 & 5 & 10 & 9 & 8 \\
\hline Unentschiedene Stellungnahme zu Religion als Grenze der MF & 2 & 3 & 0 & - & 1 & 1 \\
\hline Unentschiedene Stellungnahme zu einer sonstigen Grenze der MF & 3 & 1 & 0 & 5 & 4 & 3 \\
\hline Zensur & 14 & 4 & 1 & 7 & 5 & 6 \\
\hline Bedrohung von Medienschaffenden & 15 & - & 2 & 2 & 3 & 6 \\
\hline Sonstige Bedrohung der MF & 8 & 4 & 2 & 7 & 4 & 5 \\
\hline MF ist schutzwürdig & 3 & 3 & 17 & 10 & 9 & 9 \\
\hline Sonstiger Argumentinhalt & 30 & 49 & 36 & 22 & 26 & 32 \\
\hline Gesamt & 100 & 100 & 100 & 100 & 100 & 100 \\
\hline
\end{tabular}

$\mathrm{N}=740$ (Argumente nach Bündelung). Cramer-V=.23, Irrtumswahrscheinlichkeit $\mathrm{p}<.01$

$0 \%$ bedeutet, dass ein Argument in weniger als $1 \%$ der Fälle vorkommt; ,-“"bedeutet, dass das Argument überhaupt nicht auftaucht.

a Auch Argumente, in denen der Urheber Religion/Sonstiges als Grenze der MF ansieht und die Beschränkung einfordert, weil sie (noch) nicht umgesetzt ist.

${ }^{\mathrm{b}}$ Auch Argumente, in denen der Urheber Religion/Sonstiges selbst nicht als Grenze der MF ansieht und die Aufhebung dieser Beschränkung einfordert, weil sie von anderen so anerkannt wird. 


\subsection{Argumentationsweisen in den Artikeln}

Mit Hilfe einer Clusteranalyse über die verschiedenen Argumente innerhalb eines Artikels wurden fünf typische Argumentationsmuster identifiziert (vgl. Tab. 3).

Artikel im ersten Cluster $(n=51)$ befürworten überdurchschnittlich häufig Begrenzungen der Meinungs- und Medienfreiheit. Alle Artikel des Clusters enthalten mindestens ein Argument, das Religion als Schranke der Meinungs- und Medienfreiheit bestätigt. Über die Hälfte nennen außerdem weitere Grenzen. Dieser Argumentationstyp wird deshalb „Grenzbefürwortung“ genannt. So werden in der Süddeutschen Zeitung im Rahmen eines Berichts über gewalttätige Ausschreitungen gegen christliche Kirchen wegen der Mohammed-Karikaturen mehrere Akteure zitiert, welche die Zulässigkeit freier Äußerungen in Frage stellen: „Gläubige dürfen nicht in ihren religiösen Gefühlen verletzt und zum Objekt von Provokationen werden.“ Und: „Pressefreiheit bedeute keine Blankoerlaubnis zur Beleidigung anderer Völker und Religionen.“ (o. A. 2006d:

7) In geringem Maße werden Argumente gegen Beschränkungen gegenübergestellt. Das zweite Argumentationsmuster ist das der „Grenzenablehnung“ $(n=43)$. Hier finden sich vor allem Artikel mit Argumenten gegen die Begrenzung der Meinungsfreiheit durch religiöse wie andere Grenzen. Diese werden häufig mit Argumenten gegen Beschränkungen kontrastiert. Ein typisches Beispiel ist der Kommentar in der tageszeitung vom 2. Februar 2006, in dem es heißt: „Darf ein Medium religiöse Symbole satirisch aufgreifen? Auch wenn dies möglicherweise die Gefühle von Glaubenden verletzt? Selbstverständlich. Wo Meinungs-, Presse-, und Kunstfreiheit herrscht, ist nichts und niemand vor Satire sicher.“ Und später weiter: „Meinungsfreiheit beinhaltet auch das Recht auf Religionskritik.“ (Mika 2006: 1)

Das dritte Argumentationsmuster ist mit 53 Fällen das größte und umfasst Hinweise auf die Bedrohung von Medienschaffenden und andere Gefahren für die Meinungsfreiheit. 
Beispielsweise berichtet die Frankfurter Allgemeine Zeitung am 7. Februar 2006 (Lucius 2006: 40) unter der Überschrift „Meinungsfreiheit unter Polizeischutz: Die ,JyllandsPosten’ ist im Ausnahmezustand“"von Morddrohungen gegen die Karikaturisten sowie Bombendrohungen in Redaktionsgebäuden, und der Chefredakteur der Jyllands-Posten erklärt den Kampf um die Meinungsfreiheit für verloren.

Alle 33 Artikel des vierten Clusters erwähnen Zensurmaßnahmen. Zum Beispiel in der tageszeitung: „Wegen Abdrucks von Mohammed-Karikaturen hat die saudische Regierung eine Zeitung geschlossen. Das Informationsministerium habe das Blatt auf unbestimmte Zeit verboten [....].“ (o. A. 2006e: 9) Oder: „Malaysia verhängte eine Zensur von Zeichnungen.“ (o. A. 2006c: 3). Andere Argumente kommen in diesen Artikeln kaum vor.

Alle Artikel im fünften Cluster $(n=28)$ enthalten eine „SchutzwürdigkeitsArgumentation“. So wird in der Süddeutschen Zeitung der dänische Regierungschef Rasmussen erwähnt, der für die Freiheit der Presse kämpft (Fischer 2006: 2), oder in der Frankfurter Allgemeinen Zeitung machen deutsche Politiker, ,ganz deutlich, daß wir zur Pressefreiheit stehen“ (o. A. 2006b: 2) und „daß es eine unabhängige, durch staatliche Eingriffe nicht zu zensierende Presse geben muss [...]“ (o. A. 2006a: 4). Darüber hinausgehende Argumente sind in diesem Argumentationsmuster selten. 
Tab. 3: Anteile der Argumentinhalte nach Argumentationsclustern der Artikel, in \%

\section{Argumentationstypen}

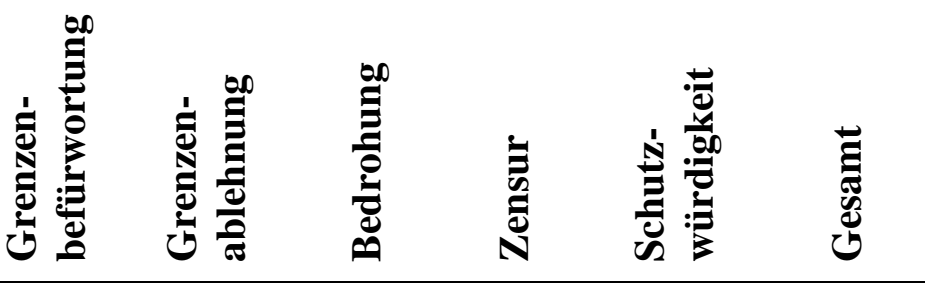

\begin{tabular}{|c|c|c|c|c|c|c|}
\hline Argumentinhalte & $(n=51)$ & $(n=43)$ & $(n=53)$ & $(n=33)$ & $(\mathbf{n}=\mathbf{2 8})$ & $(\mathrm{n}=\mathbf{2 0 8})$ \\
\hline $\begin{array}{l}\text { Befürwortung der Beschränkung der } \\
\text { MF durch Religion }{ }^{\text {a }}\end{array}$ & 100 & 44 & 0 & 15 & 14 & 38 \\
\hline $\begin{array}{l}\text { Befürwortung der Beschränkung der } \\
\text { MF durch eine sonstige Grenze }\end{array}$ & 57 & 35 & 30 & 24 & 21 & 36 \\
\hline $\begin{array}{l}\text { Ablehnung der Beschränkung der MF } \\
\text { durch Religion }^{\mathrm{b}}\end{array}$ & 4 & 47 & 4 & 9 & 0 & 13 \\
\hline $\begin{array}{l}\text { Ablehnung der Beschränkung der MF } \\
\text { durch eine sonstige Grenze }\end{array}$ & 14 & 81 & 13 & 9 & 4 & 25 \\
\hline $\begin{array}{l}\text { Unentschiedene Stellungnahme zu } \\
\text { Religion als Grenze der MF }{ }^{1}\end{array}$ & 2 & 9 & 9 & 0 & 0 & 5 \\
\hline $\begin{array}{c}\text { Unentschiedene Stellungnahme zu } \\
\text { einer sonstigen Grenze der MF }\end{array}$ & 8 & 14 & 15 & 0 & 0 & 9 \\
\hline Zensur & 4 & 0 & 2 & 100 & 11 & 19 \\
\hline Bedrohung von Medienschaffenden & 20 & 16 & 38 & 6 & 0 & 19 \\
\hline Sonstige Bedrohung der MF & 10 & 12 & 28 & 15 & 7 & 15 \\
\hline MF ist schutzwürdig & 33 & 9 & 6 & 6 & 100 & 26 \\
\hline
\end{tabular}

$\mathrm{N}=208$; Artikel, die nur sonstige Argumente enthalten, ausgeschlossen

Hierarchische Clusteranalyse mit Fusionierungsalgorithmus nach WARD

Proximitätsmaß: binäre quadrierte euklidische Distanz

Skala: $1=$ Argumentinhalt kommt mindestens einmal vor; $0=$ Argumentinhalt kommt nicht vor

Höchste Anteile je Zeile hervorgehoben

${ }^{1}$ ANOVA nicht signifikant bei einem Signifikanzniveau von $\mathrm{p} \leq .05$

a Auch Argumente, in denen der Urheber Religion/Sonstiges als Grenze der MF ansieht und die Beschränkung einfordert, weil sie (noch) nicht umgesetzt ist.

${ }^{\mathrm{b}}$ Auch Argumente, in denen der Urheber Religion/Sonstiges selbst nicht als Grenze der MF ansieht und die Aufhebung dieser Beschränkung einfordert, weil sie von anderen so anerkannt wird.

Lesebeispiel: 100 Prozent der Artikel, die dem Argumentationstyp 1 „Grenzbefürwortung“ folgen, beinhalten mindestens ein Argument, in dem Religion als eine Grenze der Meinungsfreiheit dargestellt wird. Von den 51 Artikeln mit dem Argumentationstyp Grenzbefürwortung beinhalten vier Prozent mindestens ein Argument, nach welchem Religion keine Grenze der Meinungsfreiheit ist. 
5.4 Diskussionsbeiträge der untersuchten Medien in Berichterstattung und Kommentierung

Die Argumentationen unterscheiden sich je nachdem, welche Stilform der Artikel hat, in dem sie vorkommen (vgl. Tab. 4). Es gibt einen mäßigen, aber hochsignifikanten Einfluss $($ Cramer- $\mathrm{V}=.28, p<.01)$ : In meinungsbetonten Stilformen werden Grenzen für die Meinungsfreiheit etwa doppelt so häufig zurückgewiesen, wie Beschränkungen befürwortet werden. Genau umgekehrt verhält es sich in Informationsartikeln, dort wird in $15 \%$ eine ablehnende und in $29 \%$ eine zustimmende Haltung gegenüber Begrenzungen eingenommen. Meinungsartikel thematisieren seltener als Informationsartikel Zensurmaßnahmen (6 \% zu $22 \%)$, andere Bedrohungen der Meinungsfreiheit kommen dagegen häufiger vor (35\% zu $22 \%)$.

Tab. 4: Argumentationscluster der Artikel nach journalistischen Stilformen

\section{Journalistische Stilform}

\begin{tabular}{|c|c|c|c|}
\hline & $\begin{array}{l}\text { Informationsbetonte } \\
\text { Stilformen }\end{array}$ & $\begin{array}{l}\text { Meinungsbetonte } \\
\text { Stilformen }\end{array}$ & Gesamt \\
\hline Argumentationscluster & $(n=138)$ & $(n=52)$ & $(n=190)$ \\
\hline Grenzenbefürwortung & 29 & 15 & 25 \\
\hline Grenzenablehnung & 15 & 29 & 19 \\
\hline Bedrohung & 22 & 35 & 25 \\
\hline Zensur & 22 & 6 & 17 \\
\hline Schutzwürdigkeit & 12 & 15 & 13 \\
\hline Gesamt & 100 & 100 & 100 \\
\hline
\end{tabular}

$\mathrm{N}=190$; Artikel, die nur sonstige Argumente enthalten, ausgeschlossen; Artikel mit nicht zuordenbaren Stilformen ausgeschlossen Cramer-V=.28, Irrtumswahrscheinlichkeit $\mathrm{p}<.01$ Informationsbetonte Artikel: Nachricht, Bericht, Lexikonartikel Meinungsbetonte Artikel: Kommentar, Kolumne, Glosse, Kritik 
Zwischen den drei untersuchten überregionalen deutschen Tageszeitungen Frankfurter Allgemeine Zeitung, Süddeutsche Zeitung und die tageszeitung konnten keine signifikanten Einflüsse der Zeitungen auf die gewählten Argumentationsmuster gefunden werden (bei $p<.05)$.

\section{Interpretation}

An der Auswertung, welchen Inhalts die vorgebrachten Argumente sind, lässt sich ablesen: Die Diskussion in den untersuchten Zeitungen konzentriert sich stark auf ihren Auslöser, denn Bezüge auf Grenzen der Meinungsfreiheit, insbesondere auf Religion, sind dominant. Teilweise weitet sich der Mediendiskurs auf andere Grenzen der Meinungsfreiheit aus. Die Ablehnung oder Befürwortung von Beschränkungen bildet typische Argumentationsmuster. Die in den drei Zeitungen untersuchte Debatte begibt sich aber selten auf eine theoretische Ebene, auf der Begründungszusammenhänge erklärt oder bewertet würden, und auch kontrastierende Gegenüberstellungen von Begrenzungen und Nicht-Beschränkung sind selten. Dafür kann es mehrere Gründe geben: Zu-

nächst ist es möglich, dass zugunsten der Laienorientierung öffentlicher Kommunikation (vgl. Berens 2001; Gerhards/Neidhardt 1991) Komplexität und Hintergründe vermieden werden und sich die Zeitungen stattdessen ausschließlich auf anschaulichere Aspekte des Konflikts beschränken. Nicht klären lässt sich freilich, ob in der ereignisorientierten Berichterstattung die theoretischen Begründungen die Selektionshürde der Zeitungen nicht überwanden oder ob die Akteure eine theoretische Auseinandersetzung überhaupt nicht führten. Eventuell liegt die Begründungsarmut deshalb an dem allgemein im Westen vorausgesetzten Konsens über die Meinungsfreiheit, die für die deutschen Journalisten und ihr Publikum scheinbar nicht grundsätzlich zur Diskussion steht. Die Diskutanten oder Journalisten sehen also unter Umständen keine Notwendigkeit, 
durch Explikation von Begründungen zu überzeugen. Gegen diese Erklärung spricht allerdings, dass Gefahren für die Meinungs- und Medienfreiheit typische Artikelinhalte bilden; die Freiheit scheint also doch nicht so sicher.

Die Häufigkeit der Teilnahme von Medienakteuren unterstützt die oben geäußerte Vermutung, dass die Medien selbst zu Sprechern werden und im Rahmen einer Diskussion über die Meinungsfreiheit diese Möglichkeit verstärkt ergreifen, weil die sie selbst direkt betrifft. Schranken und Nicht-Begrenzung der Meinungsfreiheit wogen hauptsächlich die Medienakteure ab, die anderen beteiligten Gruppen sprachen sich häufiger für Beschränkungen aus. Dies ist bemerkenswert, da Medien an einer möglichst unbegrenzten Meinungsfreiheit besonderes Interesse haben dürften. Dies könnte daran liegen, dass die Zeitungen die Aspekte ergänzen, die von den anderen selten vorgebracht werden, oder dass sie einen anderen Schwerpunkt setzen. Die Zeitungen werden damit ihrer bereits in anderen Konflikten festgestellten (vgl. Weßler 1999) Funktion als Vermittler zwischen Koalitionen gerecht. Die Politik stellte zwar die Schutzwürdigkeit der Freiheit heraus, Begründungen wurden aber auch hier nicht geliefert. Anscheinend argumentieren Politiker stärker medienbezogen und orientieren sich an der einfachen und eindeutigen Struktur einer Konfliktberichterstattung. Vielleicht selektieren die Zeitungen auch einseitig, was ihrer eigenen abwägenden Haltung allerdings widersprechen würde.

Die Journalisten beziehen unterschiedliche Haltungen, je nachdem, ob sie sich im Informations- oder Meinungsteil der Zeitung äußern. Wenn sie ihre eigene Meinung kundtun können, ohne neutral vermitteln zu müssen, machen sie deutlich, dass sie Beschränkungen ihrer eigenen Arbeit zugunsten anderer Werte eher ablehnen, und verteidigen ihre Schaffensfreiheit - eine Position, die durchaus plausibel ist. Während Kommentare sich häufig Gefahren für die Meinungsfreiheit wie Bedrohungen von Medien- 
schaffenden zuwenden, finden Akte der Zensur deutlich weniger Platz im Meinungsteil der Zeitung. Scheinbar nutzen die Journalisten Kommentare lieber, um ihre persönliche Gefährdung auszuführen, Informationen über Zensuren werden dagegen sachlich vermittelt.

Die Argumentationsmuster in den drei untersuchten Zeitungen Frankfurter Allgemeine Zeitung, Süddeutsche Zeitung und die tageszeitung unterschieden sich nicht signifikant. Es kann daher vermutet werden, dass das Thema Meinungsfreiheit über redaktionellen Tendenzen steht. Die Journalisten dürften also zuerst Medienschaffende sein - dies ist die Position, von der aus sie sich an der Debatte beteiligen - und erst an zweiter Stelle andere individuelle oder politische Interessen verfolgen, die im Karikaturenstreit hinter der Verteidigung der Meinungsfreiheit zurückstehen müssen.

\section{Fazit}

Der Konflikt um die Mohammed-Karikaturen zeigt deutlich: Wie ein angemessener Ausgleich zwischen dem Nutzen und den Kosten der Meinungsfreiheit, zwischen ihren Möglichkeiten und ihren Grenzen erreicht werden kann, muss immer wieder neu ausgehandelt werden. Eine solche Diskussion kann dazu dienen, sich die eigenen Werte neu vor Augen zu führen und gegebenenfalls zu überdenken. Denn „die sozialen Spannungen und die aufeinanderprallenden Interessen im öffentlichen Raum [bilden] gerade das produktive (Zukunftslösungs-) Potential eines republikanisch verfassten Staates. [...] Das Austragen von Konflikten ist ein gesellschaftliches Therapeutikum, auch wenn es vielen unerträglich erscheint.“ (Ginthum 1999: letzter Abs.) Die Abwägung muss aber sowohl Chancen als auch Risiken der Meinungsfreiheit berücksichtigen und die dahinterliegenden Begründungen kennen. Für die untersuchen Zeitungen muss festgestellt werden, dass im Rahmen des Karikaturenstreits nur in geringem Umfang eine vielseiti- 
ge Diskussion über die Meinungsfreiheit stattgefunden hat. Die Gewinne, welche die Meinungsfreiheit bietet, waren argumentativ kaum präsent. Zwar stellten die Zeitungen ihre Grenzen ausführlich in Frage, unterstrichen ihre Schutzwürdigkeit und informierten über Bedrohungen, der Grund für ihre Notwendigkeit wurde aber kaum angesprochen. Eine solch oberflächliche Betrachtung ist dem wichtigen Gegenstand nicht angemessen und leistet einer Gefährdung der Meinungsfreiheit Vorschub. Gewiss darf davon ausgegangen werden, dass die am öffentlichen Diskurs beteiligten Akteure, Medienschaffende und Politiker, Kenntnis über die Begründungen der Meinungs- und Medienfreiheit haben. Inwiefern dies aber auch für die allgemeine Bevölkerung gilt, wenn das Thema nicht einmal in dem wegen des aktuellen Konflikts um die Karikaturen besonders umfangreichen Diskurs auftaucht, ist unsicher und lässt die Begründungsarmut hochproblematisch erscheinen. Denn es gilt für die Meinungsfreiheit, was Mill 1859 in seiner Abhandlung über die Freiheit des Denkens und Redens feststellte: „Wenn für die Pflege der Erkenntnis irgend etwas wesentlicher ist als alles andere, so sicherlich die Einsicht in die Gründe der eigenen Meinungen.“ (Mill 1973: 162-163) Diese Feststellung ist nicht nur für die Meinungsfreiheit, sondern für das demokratische System insgesamt von Bedeutung. In einem demokratischen Staat muss über die Gründe für die einzelnen Grundrechte diskutiert werden, diese müssen im Konfliktfall ausführlich gegeneinander abgewogen werden, und nur mit Hilfe dieser Diskussion können die Grundrechte gesichert werden.

$\mathrm{Zu}$ berücksichtigen ist allerdings, dass lediglich deutsche überregionale Tageszeitungen untersucht wurden. Gegebenenfalls lassen sich in anderen Zeitungen mit besonders umfangreicher Hintergrundberichterstattung, in religiös orientierten Medien oder Boulevardblättern abweichende Ergebnisse finden. Ein Vergleich mit der Berichterstattung in anderen westlich geprägten Ländern kann die Diskussion in deutschen Zeitungen zu 
der Berichterstattung in anderen Ländern ins Verhältnis setzen. Da die Auseinandersetzung über die Karikaturen zwischen zwei Kulturen entstand, die Religion und Meinungsfreiheit unterschiedlich beurteilen, versteht es sich von selbst, dass ein Vergleich mit islamisch geprägten Medien interessante Ergebnisse liefern kann. 


\section{Literatur}

Amirthalingam, Kumaralingam, 2007: Free speech and religious sensitivity, in: Media Culture Society (3) 29, 509-516.

Berens, Harald, 2001: Prozesse der Thematisierung in publizistischen Konflikten.

Ereignismanagement, Medienresonanz und Mobilisierung der Öffentlichkeit am Beispiel von Castor und Brent Spar. Wiesbaden.

Bölke, Dorothee, 2000: „Das Gesetz allein kann nicht für Anstand sorgen“. Der Presserat an der Schnittstelle von Ethik und Recht, in: Gerhardt, Rudolf/Pfeifer, Hans-Wolfgang (Hrsg.), Wer die Medien bewacht. Medienfreiheit und ihre Grenzen im internationalen Vergleich. Frankfurt/Main, 43-51.

Bundesverfassungsgericht, 1952: Urteil des Ersten Senats vom 23. Oktober 1952.

Aktenzeichen 1 BvB 1/51 (SRP-Verbot). BVerfGE 2, 1.

Bundesverfassungsgericht, 1958: Urteil des Ersten Senats vom 15. Januar 1958.

Aktenzeichen 1 BvR 400/51 (Lüth). BVerfGE 7, 198.

Bundesverfassungsgericht, 1975: Beschluss des Zweiten Senats vom 29. Oktober 1975. Aktenzeichen 2 BvE 1/75. BVerfGE 40, 287.

Debatin, Bernhard,2007a: The cartoon debate and the pathologies of the global information society, in: Debatin, Bernhard (Hrsg.), Der Karikaturenstreit und die Pressefreiheit. Berlin, 13-21.

Debatin, Bernhard, 2007b: Der Karikaturenstreit und die Pressefreiheit. Berlin.

Denninger, Erhard, 1977: Freiheitlich demokratische Grundordnung. Materialien zum Staatsverständnis und zur Verfassungswirklichkeit in der Bundesrepublik. 1. und 2. Teil. Frankfurt/Main.

Deutscher Presserat, 2006: Publizistische Grundsätze (Pressekodex). Richtlinien für die publizistische Arbeit nach Empfehlungen des Deutschen Presserats. 
Beschwerdeordnung, in: http://www.presserat.de/uploads/media/Kodex_02.pdf [3.8.2006]

Eisel, Stephan, 1986: Minimalkonsens und freiheitliche Demokratie. Eine Studie zur Akzeptanz der Grundlagen demokratischer Ordnung in der Bundesrepublik Deutschland. Paderborn.

EGMR (Europäischer Gerichtshof für Menschenrechte), 2004: Beschwerde-Nr. 59320/00, 24. Juni 2004. EGMR NJW 2004, 2647ff.

Fischer, Gerhard, 2006: Rasmussens Wandel, in: Süddeutsche Zeitung vom 4.2.

Gerhards, Jürgen/Neidhardt, Friedhelm, 1991: Strukturen und Funktionen moderner Öffentlichkeit: Fragestellungen und Ansätze, in: Müller-Doohm, Stefan/NeumannBraun, Klaus (Hrsg.), Öffentlichkeit, Kultur, Massenkommunikation. Oldenburg, $31-82$.

Ginthum, Felix, 1999: Der Mißbrauch mit dem Mißbrauch. Art. 18., die Verwirkung von Grundrechten und die grundrechtsimmanente Mißbrauchsschranke, in: http://www.forum-recht-online.de/1999/499/499ginthum.htm [19.2.2007].

Grimm, Dieter, 1995: Die Meinungsfreiheit in der Rechtsprechung des Bundesverfassungsgerichts, in: Neue Juristische Wochenschrift (27) 48, 1697 1705.

Hammans, Peter, 1987: Das politische Denken der neuen Staatslehre in der Bundesrepublik. Eine Studie zum politischen Konservatismus juristischer Gesellschaftstheorie. Opladen.

Kepplinger, Hans M., 1998: Die Demontage der Politik in der Informationsgesellschaft. Freiburg.

Kepplinger, Hans M., 1994: Publizistische Konflikte. Begriffe, Ansätze, Ergebnisse, in: Neidhardt, Friedhelm (Hrsg.), Öffentlichkeit, öffentliche Meinung, soziale 
Bewegungen (Sonderheft 34 der Kölner Zeitschrift für Soziologie und Sozialpsychologie). Opladen, 214-233.

Kunelius, Risto/Eide, Elisabeth/Hahn, Oliver/Schroeder, Roland (Hrsg.), 2007: Reading the Mohammad cartoons controversy. An international analysis of press discourses on free speech and political spin. Bochum.

Lucius, Robert von, 2006: Wir haben den Kampf verloren. Meinungsfreiheit unter Polizeischutz: Die „Jyllands-Posten“ ist im Ausnahmezustand, in: Frankfurter Allgemeine Zeitung vom 7.2.

Marcic, R., 1986: Die Reichweite der Pressefreiheit, in: Langenbucher, Wolfgang $R$. (Hrsg.), Politische Kommunikation. Grundlagen, Strukturen, Prozesse. Wien, 6-7. Mathes, René, 1987: Der publizistische Konflikt um das Moderationsverbot für Franz Alt. Eine empirische Studie zur öffentlichen Konfliktkommunikation, in: Publizistik 32, 166-179.

Mathes, Rainer/Czaplicki, Andreas, 1993: Meinungsführer im Mediensystem: „Topdown“- und „Bottom-up“-Prozesse, in: Publizistik 38, 153-166.

Matthes, Jörg/Kohring, Matthias, 2004: Die empirische Erfassung von Medien-Frames, in: Medien \& Kommunikationswissenschaft 52, 56-75.

Mathes, Rainer/Pfetsch, Barbara, 1991: The role of the alternative press in the agendabuilding Process: Spill-over effects and media opinion leadership, in: European Journal of Communication 6, 33-62.

Mill, John S., 1973: Die Freiheit. Darmstadt (zuerst 1859).

Milton, John, 1984: Aeropagitica. Eine Rede für die Freiheit der Presse. An das Parlament von England (1644), in: Wilke, Jürgen (Hrsg.), Pressefreiheit. Darmstadt, 57-113.

Mika, Bascha, 2006: Meinung, Freiheit, falsche Freunde, in: die tageszeitung vom 2.2. 
o. A., 2006a: „Pressefreiheit hat Voraussetzungen“. Lammert: Bilderstreit zeigt Dringlichkeit einer Leitkulturdebatte, in: Frankfurter Allgemeine Zeitung vom 8.2.

o. A., 2006b: „Keine Entschuldigung“. FDP und Grüne werben im Karikaturenstreit für Pressefreiheit, in: Frankfurter Allgemeine Zeitung vom 9.2.

o. A., 2006c: Steine des Anstoßes, in: die tageszeitung vom 11.2.

o. A., 2006d: Aufgebrachte Muslime setzen Kirchen in Pakistan in Brand, in:

Süddeutsche Zeitung vom 21.2.

o. A., 2006e: Saudische Zeitung dicht, in: die tageszeitung vom 22.2.

Popper, Karl, 1980: Die offene Gesellschaft und ihre Feinde 2. Falsche Propheten. München.

Pöttker, Horst, 2007: Öffentlichkeit kann wichtiger sein als religiöses Empfinden, in: Debatin, Bernhard (Hrsg.), Der Karikaturenstreit und die Pressefreiheit. Berlin, 73-90.

Ricker, Reinhart, ${ }^{7}$ 2000: Medienrecht, in: Noelle-Neumann, Elisabeth/Schulz, Winfried/Wilke, Jürgen (Hrsg.), Das Fischer Lexikon Publizistik Massenkommunikation. Frankfurt/Main, 244-267.

Ronneberger, Franz, 1977: Legitimation durch Öffentlichkeit. Düsseldorf.

Siebert, Fred S./Peterson, Theodore/Schramm, Wilbur, 1963: Four theories of the press. The authoritarian, liberitarian, social responsibility and soviet communist concept of what the press should be and do. Urbana/Chicago.

Weiß, Hans-Jürgen, 1989: Öffentliche Streitfragen und massenmediale Argumentationsstrukturen. Ein Ansatz zur Analyse der inhaltlichen Dimension im Agenda Setting-Prozeß, in: Kaase, Max/Schulz, Winfried (Hrsg.), 
Massenkommunikation. Theorien, Methoden, Befunde (Sonderheft 30 der Kölner Zeitschrift für Soziologie und Sozialpsychologie). Opladen, 473-489. Weßler, Hartmut, 1999: Öffentlichkeit als Prozeß. Deutungsstrukturen und Deutungswandel in der deutschen Drogenberichterstattung. Opladen. Wilke, Jürgen, 1987: Leitideen in der Begründung der Pressefreiheit, in: Bobrowsky, Manfred/Duchkowitsch, Wolfgang/Haas, Hannes (Hrsg.), Medien- und Kommunikationsgeschichte. Ein Textbuch zur Einführung. Wien, 92-104.

Teresa K. Naab M.A. ist wissenschaftliche Mitarbeiterin am Institut für Journalistik und Kommunikationsforschung der Hochschule für Musik und Theater Hannover

Prof. Dr. Helmut Scherer ist Professor für Kommunikations- und Medienwissenschaft am Institut für Journalistik und Kommunikationsforschung der Hochschule für Musik und Theater Hannover 